\title{
A subdifferential characterization of Motzkin decomposable functions
}

\author{
J. E. Martínez-Legaz \\ Departament d'Economia i d'Història Econòmica \\ Universitat Autònoma de Barcelona \\ and Barcelona Graduate School of Mathematics (BGSMath) \\ Spain \\ L. Thibault \\ Institut Montpelliérain Alexander Grothendieck \\ Université de Montpellier \\ France
}

May 24, 2017

\begin{abstract}
The paper provides a new subdifferential characterization for Motzkin decomposable (convex) functions. This characterization leads to diverse stability properties for such a decomposability for operations like addition and composition.
\end{abstract}

Keywords: Motzkin decomposition, recession cone, subdifferential, LegendreFenchel conjugate.

\section{Introduction}

T. Motzkin proved in his 1936 paper [5] that any polyhedral convex set in $\mathbb{R}^{n}$ can be written as the (Minkowski) sum of a polytope and a polyhedral convex cone. Based on this result, subsets in $\mathbb{R}^{n}$ which can be decomposed as the sum of a convex compact set and a closed convex cone have been studied in great detail in [1], where the name of Motzkin decomposable have been coined for such sets. Various characterizations and properties of Motzkin decomposable sets are established in [1, 2, 3, 4]. In [1] Motzkin decomposable functions are introduced as functions whose epigraphs are Motzkin decomposable sets, and this paper puts in light many properties of such functions.

The aim of the present paper is to provide a new characterization of Motzkin decomposable functions via their subdifferentials and to derive certain stability results related to some operations. In Section 2 we recall diverse basic notions 
and features in Convex Analysis which are utilized through the paper. Section 3 is devoted to the subdifferential characterization of the Motzkin decomposability for convex functions. In the last section we prove the results on the stability of this property for functions, when operations like addition and composition are concerned.

\section{Preliminaries}

Throughout $\langle\cdot, \cdot\rangle$ will denote the usual Euclidean inner product on $\mathbb{R}^{n}$ and $\|\cdot\|$ the Euclidean associated norm, and $\mathbb{B}$ will stand for the closed unit ball centered at the origin. If $S$ is a subset of $\mathbb{R}^{n}$, its closure (resp. convex hull) will be denoted by $\operatorname{cl} S$ (resp. $\operatorname{co} S)$.

Given an extended real-valued function $f: \mathbb{R}^{n} \rightarrow \mathbb{R} \cup\{-\infty,+\infty\}$, we recall that its (effective) domain $\operatorname{dom} f$ and its epigraph epi $f$ are the sets

$\operatorname{dom} f:=\left\{x \in \mathbb{R}^{n}: f(x)<+\infty\right\} \quad$ and $\quad$ epi $f:=\left\{(x, \alpha) \in \mathbb{R}^{n} \times \mathbb{R}: f(x) \leq \alpha\right\}$.

The function $f$ is said to be proper whenever it is finite at some point and does not take the value $-\infty$. The Legendre-Fenchel conjugate $f^{*}: \mathbb{R}^{n} \rightarrow$ $\mathbb{R} \cup\{-\infty,+\infty\}$ is defined by

$$
f^{*}\left(x^{*}\right):=\sup _{x \in \mathbb{R}^{n}}\left(\left\langle x, x^{*}\right\rangle-f(x)\right) \quad \text { for all } x^{*} \in \mathbb{R}^{n},
$$

and the supremum can be equivalently taken over $x \in \operatorname{dom} f$. Clearly, $f^{*}$ is a lower semicontinuous (lsc, for short) convex function. Further, when $f$ is a proper lsc convex function, it is well-known that so is $f^{*}$ and $\left(f^{*}\right)^{*}=f$.

If the function $f$ is convex, its subdifferential $\partial f(x)$ at a point $x$ where it is finite is the set

$$
\partial f(x):=\left\{x^{*} \in \mathbb{R}^{n}:\left\langle y-x, x^{*}\right\rangle \leq f(y)-f(x), \forall y \in \mathbb{R}^{n}\right\},
$$

or otherwise stated

$$
x^{*} \in \partial f(x) \Leftrightarrow\left\langle x, x^{*}\right\rangle=f(x)+f^{*}\left(x^{*}\right) .
$$

So, when $f$ is in addition proper and lsc, one sees that $x^{*} \in \partial f(x) \Leftrightarrow x \in$ $\partial f^{*}\left(x^{*}\right)$. It is also worth pointing out that $\partial f(x) \neq \emptyset$ whenever the convex function $f$ is finite at $x$ and continuous at $x$ (see, e.g., [6]).

Given two convex functions $f, g: \mathbb{R}^{n} \rightarrow \mathbb{R} \cup\{+\infty\}$, their infimal convolution $f \square g$ given by

$$
(f \square g)(x):=\inf _{y \in \mathbb{R}^{n}}(f(y)+g(x-y)) \quad \text { for all } x \in \mathbb{R}^{n},
$$

is a convex function, and clearly $\operatorname{dom} f \square g=\operatorname{dom} f+\operatorname{dom} g$. When in addition one function is continuous at a point in $\operatorname{dom} f \cap \operatorname{dom} g$, it is known (see, e.g., [6]) that $f^{*} \square g^{*}$ is a proper lsc convex function and

$$
(f+g)^{*}=f^{*} \square g^{*} \quad \text { and } \quad \partial(f+g)(x)=\partial f(x)+\partial g(x), \forall x \in \mathbb{R}^{n} .
$$


by

Given a subset $S \subset \mathbb{R}^{n}$, its indicator function $\delta_{S}: \mathbb{R}^{n} \rightarrow \mathbb{R} \cup\{+\infty\}$ is defined

$$
\delta_{S}(x)=0 \text { if } x \in S \quad \text { and } \quad \delta_{S}(x)=+\infty \text { if } x \in \mathbb{R}^{n} \backslash S .
$$

Then, the conjugate $\delta_{S}^{*}$ coincides with the support function of $S$ since

$$
\delta_{S}^{*}\left(x^{*}\right)=\sup _{x \in S}\left\langle x^{*}, x\right\rangle \quad \text { for all } x^{*} \in \mathbb{R}^{n}
$$

so $\delta_{S}^{*}(\cdot) \leq \delta_{S^{\prime}}^{*}(\cdot)$ if and only if $\operatorname{clcos} C \subset \operatorname{clco} S^{\prime}$.

It is also worth noticing that, whenever $S \neq \emptyset$, the equality $\delta_{S}^{*}(0)=0$ ensures that $\delta_{S}^{*}$ is a proper lsc convex function. Further, $\operatorname{dom} \delta_{S}^{*}$ is clearly a convex cone containing zero.

If $S$ is a nonempty closed convex set, its recession cone $0^{+} S$ is defined by

$$
0^{+} S=\left\{y \in \mathbb{R}^{n}: S+\lambda y \subset S, \forall \lambda \geq 0\right\} .
$$

The (negative) polar cone $P^{o}$ of a nonempty cone $P \subset \mathbb{R}^{n}$ is defined by

$$
P^{o}:=\left\{u \in \mathbb{R}^{n}:\langle x, u\rangle \leq 0, \forall x \in P\right\}
$$

Under the assumption that $S$ is nonempty, closed and convex, it is known (cf, e.g. [6, Corollary 14.2.1]) that

$$
0^{+} S=\left(\operatorname{dom} \delta_{S}^{*}\right)^{o}
$$

which can be also directly seen from the equality

$$
S=\left\{x \in \mathbb{R}^{n}:\left\langle x, x^{*}\right\rangle \leq \delta_{S}^{*}\left(x^{*}\right), \forall x^{*} \in \operatorname{dom} \delta_{S}^{*}\right\} .
$$

We recall now the concepts of Motzkin decomposable sets and functions.

Definition 1 A nonempty closed convex set $S$ in $\mathbb{R}^{n}$ is said to be Motzkin decomposable (M-decomposable, for short) provided that there are a compact convex set $K$ in $\mathbb{R}^{n}$ and a closed convex cone $P$ in $\mathbb{R}^{n}$ such that $S=K+P$ (the sum being taken in Minkowski's sense). Such an equality is called a Motzkin representation (or decomposition) of $S$ with compact and conic components $K$ and $P$, respectively.

A proper lsc convex function $f: \mathbb{R}^{n} \rightarrow \mathbb{R} \cup\{+\infty\}$ is then called Motzkin decomposable (M-decomposable, for short) when its epigraph epi $f$ is an $M$ decomposable set in $\mathbb{R}^{n+1}$.

The following theorem recalls in another equivalent form the result of Proposition 16 in [1]; it will be crucial in the next section.

Theorem 2 A nonempty closed convex set $S$ in $\mathbb{R}^{n}$ is $M$-decomposable if and only if there exists a compact convex set $K \subset S$ such that for each $x^{*} \in \operatorname{dom} \delta_{S}^{*}$ there exists some $\bar{x} \in K$ such that $\delta_{S}^{*}\left(x^{*}\right)=\left\langle\bar{x}, x^{*}\right\rangle$ (in other words, $\operatorname{dom} \delta_{S}^{*}=$ $\left.\partial \delta_{S}(K)\right)$. 


\section{Subdifferential characterization of M-decomposable functions}

Theorem 3 A proper lsc convex function $f: \mathbb{R}^{n} \rightarrow \mathbb{R} \cup\{+\infty\}$ is M-decomposable if and only if there exists a compact convex set $K \subset \operatorname{dom} f$ such that $\operatorname{cldom} f=$ $K+0^{+}(\operatorname{cl} \operatorname{dom} f)$ (that is, $K$ is a compact component of $\left.\mathrm{cldom} f\right), f$ is bounded on $K$, and $\partial f(K)=\operatorname{dom} f^{*}$.

Proof. Assume first that $f$ is M-decomposable, i.e., that epi $f$ is an M-decomposable set. Then, by Theorem 2 there exists a compact set $C \subset$ epif such that for every $\left(x^{*}, \alpha^{*}\right) \in \operatorname{dom} \delta_{\text {epi } f}^{*}$ there exists $(x, \alpha) \in C$ satisfying

$$
\delta_{\text {epif }}^{*}\left(x^{*}, \alpha^{*}\right)=\left\langle x, x^{*}\right\rangle+\alpha \alpha^{*} .
$$

Let $\widehat{C}$ be the projection of $C$ onto $\mathbb{R}^{n}$, that is,

$$
\widehat{C}:=\left\{x \in \mathbb{R}^{n}:(x, \alpha) \in C \text { for some } \alpha \in \mathbb{R}\right\} .
$$

Clearly, $K:=\operatorname{co} \widehat{C}$ is a compact convex subset of $\operatorname{dom} f$. We will now prove that cldom $f=K+0^{+}(\operatorname{cldom} f)$. Let $x^{*} \in \operatorname{dom} \delta_{\text {cldom } f}^{*}$. Since

$$
\delta_{\text {epi } f}^{*}\left(x^{*}, 0\right)=\delta_{\operatorname{dom} f}^{*}\left(x^{*}\right)=\delta_{\operatorname{cldom} f}^{*}\left(x^{*}\right),
$$

we have $\left(x^{*}, 0\right) \in \operatorname{dom} \delta_{\text {epi } f}^{*}$; hence there exists $(\bar{x}, \alpha) \in C$ satisfying

$$
\left\langle\bar{x}, x^{*}\right\rangle=\delta_{\text {epi } f}^{*}\left(x^{*}, 0\right)=\delta_{\text {cldom } f}^{*}\left(x^{*}\right) .
$$

Since $\bar{x} \in \widehat{C} \subset K$, we deduce that $\delta_{\text {cldom } f}^{*}\left(x^{*}\right) \leq \delta_{K}^{*}\left(x^{*}\right)$. This being true for any $x^{*} \in \operatorname{dom} \delta_{\operatorname{cldom} f}^{*}$, it ensues by lower semicontinuity of $\delta_{\mathrm{cldom} f}^{*}$ and by continuity of $\delta_{K}^{*}$ on $\mathbb{R}^{n}$ that $\delta_{\operatorname{cldom} f}^{*}\left(x^{*}\right) \leq \delta_{K}^{*}\left(x^{*}\right)$ for all $x^{*} \in \operatorname{cl}\left(\operatorname{dom} \delta_{\operatorname{cldom} f}^{*}\right)$, which entails that $\operatorname{cl}\left(\operatorname{dom} \delta_{\operatorname{cldom} f}^{*}\right) \subset \operatorname{dom} \delta_{\operatorname{cldom} f}^{*}$. Then, $\operatorname{dom} \delta_{\operatorname{cldom} f}^{*}$ is closed and

$$
\delta_{\operatorname{cldom} f}^{*}\left(x^{*}\right) \leq \delta_{K}^{*}\left(x^{*}\right) \leq \delta_{K+0^{+}(\operatorname{cldom} f)}^{*}\left(x^{*}\right) .
$$

On the other hand, if $x^{*} \notin \operatorname{dom} \delta_{\operatorname{cldom} f}^{*}$, we have $x^{*} \notin\left(0^{+}(\operatorname{cldom} f)\right)^{o}$ by $(3)$, and hence $\delta_{K+0^{+}(\operatorname{cldom} f)}^{*}\left(x^{*}\right)=+\infty$ (as easily seen), so the latter inequality

$$
\delta_{\mathrm{cl} \operatorname{dom} f}^{*}\left(x^{*}\right) \leq \delta_{K+0^{+}(\operatorname{cldom} f)}^{*}\left(x^{*}\right)
$$

still holds true. We derive that $\operatorname{cldom} f \subset K+0^{+}(\operatorname{cldom} f)$, and the inclusion is in fact an equality because

$$
K+0^{+}(\operatorname{cldom} f) \subset \operatorname{cldom} f+0^{+}(\operatorname{cldom} f)=\operatorname{cldom} f .
$$

This justifies the desired M-decomposability of cldom $f$ with $K$ as compact component.

We will next prove that $f$ is bounded on $K$. Since $f$ is lsc and $K$ is compact, we only have to prove boundedness from above. Clearly, $f$ is bounded above on 
$\widehat{C}$ by $\max \left\{\alpha \in \mathbb{R}:(x, \alpha) \in C\right.$ for some $\left.x \in \mathbb{R}^{n}\right\}$, and by the convexity of $f$ the same upper bound applies on $K$. Let us now prove that $\partial f(K)=\operatorname{dom} f^{*}$. Since the inclusion $\subset$ obviously holds, we only need to prove the opposite inclusion. Let $x^{*} \in \operatorname{dom} f^{*}$. We have $\delta_{\text {epi } f}^{*}\left(x^{*},-1\right)=f^{*}\left(x^{*}\right) ;$ hence $\left(x^{*},-1\right) \in \operatorname{dom} \delta_{\text {epi } f}^{*}$. Therefore, by (4) there exists $(\bar{x}, \alpha) \in C$ satisfying

$$
\left\langle\bar{x}, x^{*}\right\rangle-\alpha=\delta_{\text {epi } f}^{*}\left(x^{*},-1\right)=f^{*}\left(x^{*}\right) .
$$

Using the fact that $\alpha \geq f(\bar{x})$ it readily follows that $x^{*} \in \partial f(\bar{x}) \subset \partial f(K)$, which proves the required inclusion, and hence the equality. The implication $\Rightarrow$ is then justified.

Conversely, let us now assume the existence of $K$ as in the statement of the theorem, and define

$$
a:=\min \{f(x): x \in K\}, b:=\sup \{f(x): x \in K\} \text { and } C:=K \times[a, b] .
$$

The set $C$ is obviously convex and compact. Let $\left(x^{*}, \alpha^{*}\right) \in \operatorname{dom} \delta_{\text {epi } f}^{*}$. Then $\alpha^{*} \leq 0$. If $\alpha^{*}=0$ then, as $\delta_{\text {cldom } f}^{*}\left(x^{*}\right)=\delta_{\text {epi } f}^{*}\left(x^{*}, 0\right)$, we have $x^{*} \in \operatorname{dom} \delta_{\text {cldom } f}^{*} \subset$ $\operatorname{dom} \delta_{K}^{*}$; hence there exists $\bar{x} \in K$ such that

$$
\left\langle\bar{x}, x^{*}\right\rangle=\delta_{K}^{*}\left(x^{*}\right)=\delta_{\mathrm{cldom} f}^{*}\left(x^{*}\right)=\delta_{\text {epi } f}^{*}\left(x^{*}, 0\right),
$$

where the second equality is due to the assumption $\mathrm{cl} \operatorname{dom} f=K+0^{+}(\operatorname{cl} \operatorname{dom} f)$ and to (3). We thus have

$$
\delta_{\text {epi } f}^{*}\left(x^{*}, 0\right)=\left\langle\bar{x}, x^{*}\right\rangle+a 0,
$$

and further $(\bar{x}, a) \in C$ by definition of $C$.

Suppose now that $\alpha^{*}<0$. Then, since

$$
f^{*}\left(\frac{x^{*}}{-\alpha^{*}}\right)=\delta_{\text {epi } f}^{*}\left(\frac{x^{*}}{-\alpha^{*}},-1\right)=\frac{1}{-\alpha} \delta_{\text {epi } f}^{*}\left(x^{*}, \alpha^{*}\right)
$$

we deduce that $\frac{x^{*}}{-\alpha^{*}} \in \operatorname{dom} f^{*}=\partial f(K)$, where the equality follows by assumption. Consequently, there is some $\bar{x} \in K$ such that $f^{*}\left(\frac{x^{*}}{-\alpha^{*}}\right)=\left\langle\bar{x}, \frac{x^{*}}{-\alpha^{*}}\right\rangle-f(\bar{x})$, and equivalently

$$
\delta_{\text {epi } f}^{*}\left(x^{*}, \alpha^{*}\right)=-\alpha^{*} f^{*}\left(\frac{x^{*}}{-\alpha^{*}}\right)=\left\langle\bar{x}, x^{*}\right\rangle+\alpha^{*} f(\bar{x}) ;
$$

moreover, $(\bar{x}, f(\bar{x})) \in C$ according to the definition of $C$.

We can then apply Theorem 2 to conclude that epi $f$ is M-decomposable, which means that $f$ is an M-decomposable function.

Remark 4 The "if" statement does not require convexity of $K$; in fact, one can easily check that if a compact set $K$ satisfies the stated conditions so does the compact convex set co $K$. A simple example where all the conditions in Theorem 3 are fulfilled with a nonconvex compact set $K$ is furnished, as it is not difficult 
to verify, by the compact set $K:=\{-1,1\}$ in $\mathbb{R}$ and the function $f: \mathbb{R} \rightarrow \mathbb{R}$ defined by

$$
f(x)=\max \{|x|, 1\} \quad \text { for all } x \in \mathbb{R} .
$$

Before stating a first corollary, let us establish the following lemma which has its own interest even in the setting of general normed spaces.

Lemma 5 Let $g: X \rightarrow \mathbb{R} \cup\{+\infty\}$ be a proper convex function on a normed space $X$ and $C$ be a subset of $X$ for which there exists a bounded set $Q$ in the topological dual space $X^{*}$ such that $\partial g(x) \cap Q \neq \emptyset$ for all $x \in C$. Then, the restriction of $g$ to $C$ is Lipschitz.

Proof. By assumption there exist a real $\gamma \geq 0$ with $Q \subset \gamma \mathbb{B}_{X^{*}}$ along with a mapping $\zeta: C \rightarrow X^{*}$ such that $\zeta(x) \in \partial g(x) \cap Q$ for all $x \in C$. Then, for any $x, y \in C$ we have by definition of subdifferential of a convex function

$$
g(y) \geq g(x)+\langle y-x, \zeta(x)\rangle \geq g(x)-\gamma\|y-x\|,
$$

or equivalently $g(x)-g(y) \leq \gamma\|x-y\|$, which confirms the Lipschitz property of $g$ on $C$.

Corollary 6 If a proper lsc convex function $f: \mathbb{R}^{n} \rightarrow \mathbb{R} \cup\{+\infty\}$ is $M$ decomposable, then $f^{*}$ is Lipschitz on its domain.

Proof. Let $K$ be the compact set in $\mathbb{R}^{n}$ given by Theorem 3 . By this theorem and by (1) it easily follows that $\partial f^{*}\left(x^{*}\right) \cap K \neq \emptyset$ for every $x^{*} \in \operatorname{dom} f^{*}$. Since $K$ is bounded, this condition implies by the above lemma that $f^{*}$ is Lipschitz on $\operatorname{dom} f^{*}$.

Remark 7 Consider the function $f:=\|\cdot\|+\delta_{\{1\} \times \mathbb{R}}$ on $\mathbb{R}^{2}$. For any $(u, v) \in \mathbb{R}^{2}$ we have by calculus rule for the Legendre-Fenchel conjugate of the sum of two convex functions one of which is continuous (see (2)),

$$
\begin{aligned}
f^{*}(u, v) & =\inf _{(x, y) \in \mathbb{R}^{n}}\left(\delta_{\{1\} \times \mathbb{R}}(x, y)+\|(u, v)-(x, y)\|\right) \\
& =\inf _{(x, y) \in\{1\} \times \mathbb{R}}\|(u, v)-(x, y)\|=|u-1| .
\end{aligned}
$$

On the other hand, $\partial f(1, y)=\frac{(1, y)}{\|(1, y)\|}+\mathbb{R} \times\{0\}$ for any $y \in \mathbb{R}$ (see the second equality in (2)), so there is no compact set $K$ such that $\partial f(K)=\mathbb{R}^{2}$. Since $\operatorname{dom} f^{*}=\mathbb{R}^{2}$, Theorem 3 ensures that $f$ is not $M$-decomposable, while $f^{*}$ is Lipschitz on its domain. This tells us that the converse of Corollary 6 fails.

Despite the above counterexample, the situation is different when $f$ is finitevalued on $\mathbb{R}^{n}$.

Corollary 8 Let $f: \mathbb{R}^{n} \rightarrow \mathbb{R}$ be a finite-valued convex function. Then the following statements are equivalent: 
(a) $f$ is $M$-decomposable.

(b) There exists a compact convex set $K \subset \mathbb{R}^{n}$ such that $\partial f(K)=\operatorname{dom} f^{*}$.

(c) There exists a bounded set $Q \subset \mathbb{R}^{n}$ such that $\partial f(Q)=\operatorname{dom} f^{*}$.

(d) $f^{*}$ is Lipschitz on its domain.

Proof. The equivalence between (a) and (b) is an immediate consequence of Theorem 3. Implication (b) $\Longrightarrow$ (c) is trivial, and (c) implies (b) by taking $K:=\operatorname{clco} Q$ since by (1) and (c) we have

$$
\operatorname{dom} f^{*}=\partial f(Q) \subset \partial f(K) \subset \partial f\left(\mathbb{R}^{n}\right) \subset \operatorname{dom} f^{*} .
$$

Implication (a) $\Longrightarrow$ (d) follows from Corollary 6 .

It remains to prove that (d) implies (b). Assume that $f^{*}$ is Lipschitz on its domain with some constant $\gamma \geq 0$. Put $K:=\gamma \mathbb{B}$ and note that $\partial f(K) \subset$ $\operatorname{dom} f^{*}$, since $\partial f\left(\mathbb{R}^{n}\right) \subset \operatorname{dom} f^{*}$ by (1). On the other hand, it is known (and not difficult to see) that the convex function $\varphi:=f^{*} \square(\gamma\|\cdot\|)$ is finite-valued and $\gamma$-Lipschitz on $\mathbb{R}^{n}$ and it coincides with $f^{*}$ on $\operatorname{dom} f^{*}$. Fix any $x^{*} \in \operatorname{dom} f^{*}$. By continuity of $\varphi$ we may choose some $\bar{x} \in \partial \varphi\left(x^{*}\right)$, and by the $\gamma$-Lipschitz property of $\varphi$ we have $\|\bar{x}\| \leq \gamma$ (as known and easily verified). Since $\varphi \leq f^{*}$ with $\varphi\left(x^{*}\right)=f^{*}\left(x^{*}\right)$, the definition of subdifferential implies $\partial \varphi\left(x^{*}\right) \subset \partial f^{*}\left(x^{*}\right)$, so $\bar{x} \in \partial f^{*}\left(x^{*}\right)$, or equivalently $x^{*} \in \partial f(\bar{x})$ according to (1). This ensures that $\operatorname{dom} f^{*} \subset \partial f(K)$, hence the equality $\partial f(K)=\operatorname{dom} f^{*}$ is shown, which finishes the proof of the corollary.

\section{Sum and composition}

Consider on $\mathbb{R}^{2}$ the functions $g:=\|\cdot\|$ and $h:=\delta_{\{1\} \times \mathbb{R}}$. Corollary $8(\mathrm{~d})$ tells us that $g$ is M-decomposable since $g^{*}=\delta_{\mathbb{B}}$ is Lipschitz on its domain, and the function $h$ is also M-decomposable because its epigraph

$$
\text { epi } h=\{1\} \times \mathbb{R} \times[0,+\infty[=\{(1,0,0)\}+\{0\} \times \mathbb{R} \times[0,+\infty[
$$

is an M-decomposable set. Nevertheless, the sum $g+h$ is not M-decomposable according to Remark 7.

The next proposition provides a case when the sum is M-decomposable. It has been previously established in [3, Theorem 25]. Our proof below is very simple.

Proposition 9 Let $f: \mathbb{R}^{n} \rightarrow \mathbb{R} \cup\{+\infty\}$ be a proper lsc convex function, $\alpha \in \mathbb{R}$ and $\ell: \mathbb{R}^{n} \rightarrow \mathbb{R}$ be a linear function. If $f$ is $M$-decomposable, then $f+\ell+\alpha$ is also M-decomposable.

Proof. The convex function $\varphi:=f+\ell+\alpha$ is clearly lsc and proper with $\operatorname{dom} \varphi=$ $\operatorname{dom} f$. By Theorem 3 there exists a compact convex set $K \subset \mathbb{R}^{n}$ over which $f$ is bounded and such that $\operatorname{cldom} f=K+0^{+}(\operatorname{cldom} f)$ and $\partial f(K)=\operatorname{dom} f^{*}$. 
Obviously $\varphi$ is also bounded on $K$ and $\operatorname{cldom} \varphi=K+0^{+}(\operatorname{cldom} \varphi)$. Further, as easily seen $\varphi^{*}\left(x^{*}\right)=f^{*}\left(x^{*}-y^{*}\right)-\alpha$, with $y^{*} \in \mathbb{R}^{n}$ such that $l=\left\langle\cdot, y^{*}\right\rangle$, so $\operatorname{dom} \varphi^{*}=y^{*}+\operatorname{dom} f^{*}$. From this and the above equality $\partial f(K)=\operatorname{dom} f^{*}$ (using the second equality in $(2)$ ) we see that $\partial \varphi(K)=\operatorname{dom} \varphi^{*}$. Altogether we obtain by Theorem 3 again that $\varphi$ is M-decomposable.

The case of the sum of two functions with separate variables is also of interest.

Proposition 10 Let $f: \mathbb{R}^{n} \rightarrow \mathbb{R} \cup\{+\infty\}$ and $g: \mathbb{R}^{m} \rightarrow \mathbb{R} \cup\{+\infty\}$ be two proper lsc convex functions which are $M$-decomposable. Then, the function $\varphi$ : $\mathbb{R}^{n} \times \mathbb{R}^{m} \rightarrow \mathbb{R} \cup\{+\infty\}$ defined by

$$
\varphi(x, y):=f(x)+g(y) \quad \text { for all }(x, y) \in \mathbb{R}^{n} \times \mathbb{R}^{m},
$$

is $M$-decomposable.

Proof. Suppose first that $f$ and $g$ are M-decomposable. By Theorem 3 there is a compact convex set $K_{f}$ (resp. $K_{g}$ ) in $\mathbb{R}^{n}$ (resp. $\mathbb{R}^{m}$ ) over which $f$ (resp. $g$ ) is bounded and such that $\operatorname{cldom} f=K_{f}+0^{+}(\operatorname{cldom} f)$ (resp. cldom $g=K+$ $\left.0^{+}(\operatorname{cldom} g)\right)$ and $\partial f\left(K_{f}\right)=\operatorname{dom} f^{*}\left(\operatorname{resp} . \partial g\left(K_{g}\right)=\operatorname{dom} g^{*}\right)$. The function $\varphi$ is obviously proper, lcs and convex, and it is bounded on $K_{f} \times K_{g}$. It is also easily checked from the equality $\operatorname{dom} \varphi=\operatorname{dom} f \times \operatorname{dom} g$ that

$$
\operatorname{cldom} \varphi=K_{f} \times K_{g}+0^{+}(\operatorname{cldom} \varphi) .
$$

Note also that $\varphi^{*}\left(x^{*}, y^{*}\right)=f^{*}\left(x^{*}\right)+g^{*}\left(y^{*}\right)$, so $\operatorname{dom} \varphi^{*}=\left(\operatorname{dom} f^{*}\right) \times\left(\operatorname{dom} g^{*}\right)$. Then, since $\partial \varphi(x, y)=\partial f(x) \times \partial g(y)$ (as readily verified), we have

$$
\partial \varphi\left(K_{f} \times K_{g}\right)=\partial f\left(K_{f}\right) \times \partial g\left(K_{g}\right)=\left(\operatorname{dom} f^{*}\right) \times\left(\operatorname{dom} g^{*}\right)=\operatorname{dom} \varphi^{*} .
$$

Applying Theorem 3 again, we obtain that the function $\varphi$ is M-decomposable.

Concerning Cartesian products of sets we have the following result.

Proposition 11 Let $S$ and $S^{\prime}$ be two nonempty closed convex sets in $\mathbb{R}^{n}$ and $\mathbb{R}^{m}$ respectively. Then $S \times S^{\prime}$ is $M$-decomposable in $\mathbb{R}^{n} \times \mathbb{R}^{m}$ if and only if $S$ and $S^{\prime}$ are $M$-decomposable in $\mathbb{R}^{n}$ and $\mathbb{R}^{m}$ respectively.

Proof. The implication $\Leftarrow$ is obvious. Suppose that $S \times S^{\prime}$ is M-decomposable in $\mathbb{R}^{n} \times \mathbb{R}^{m}$ and choose by definition a compact convex set $K$ in $\mathbb{R}^{n} \times \mathbb{R}^{m}$ such that

$$
S \times S^{\prime}=K+0^{+}\left(S \times S^{\prime}\right) .
$$

Denote by $C$ (resp. $C^{\prime}$ ) the projection of $K$ on $\mathbb{R}^{n}$ (resp. $\left.\mathbb{R}^{m}\right)$. The set $C \times C^{\prime}$ is convex and compact in $\mathbb{R}^{n} \times \mathbb{R}^{m}$, and clearly $C \times C^{\prime} \subset S \times S^{\prime}$ since $K \subset S \times S^{\prime}$. This and the obvious inclusion $K \subset C \times C^{\prime}$ entail that

$S \times S^{\prime}=K+0^{+}\left(S \times S^{\prime}\right) \subset C \times C^{\prime}+0^{+}\left(S \times S^{\prime}\right) \subset S \times S^{\prime}+0^{+}\left(S \times S^{\prime}\right)=S \times S^{\prime}$, 
which combined with the (readily seen) equality $0^{+}\left(S \times S^{\prime}\right)=\left(0^{+} S\right) \times\left(0^{+} S^{\prime}\right)$ yields

$$
S \times S^{\prime}=C \times C^{\prime}+\left(0^{+} S\right) \times\left(0^{+} S^{\prime}\right)=\left(C+0^{+} S\right) \times\left(C^{\prime}+0^{+} S^{\prime}\right) .
$$

This gives $S=C+0^{+} S$ and $S^{\prime}=C^{\prime}+0^{+} S^{\prime}$, so $S$ and $S^{\prime}$ are M-decomposable in $\mathbb{R}^{n}$ and $\mathbb{R}^{m}$ respectively as desired.

Let us now turn to composition with linear mappings.

Proposition 12 Let $A: \mathbb{R}^{n} \rightarrow \mathbb{R}^{m}$ be a linear mapping, $S \subset A\left(\mathbb{R}^{n}\right)$ be a nonempty closed convex set and $f: \mathbb{R}^{m} \rightarrow \mathbb{R} \cup\{+\infty\}$ be a proper lsc convex function such that $\operatorname{dom} f \subset A\left(\mathbb{R}^{n}\right)$. The following hold:

(a) If the set $S$ is $M$-decomposable, then $A^{-1}(S)$ is $M$-decomposable.

(b) If the function $f$ is $M$-decomposable, then $f \circ A$ is also $M$-decomposable.

Proof. (a) Let $K$ be a compact convex set in $\mathbb{R}^{m}$ and $P$ be a closed convex cone in $\mathbb{R}^{m}$ such that $S=K+P$. We claim that $A^{-1}(S)=A^{-1}(K)+A^{-1}(P)$. First, we note that, for any $u \in A^{-1}(K)$ and $v \in A^{-1}(P)$, we have

$$
A(u+v)=A u+A v \in K+P=S,
$$

that is, $u+v \in A^{-1}(S)$; this means that $A^{-1}(K)+A^{-1}(P) \subset A^{-1}(S)$. For the converse inclusion, fix any $x \in A^{-1}(S)$. Then, $A x=y+z$ with $y \in K$ and $z \in P$. By the inclusion $S \subset A\left(\mathbb{R}^{n}\right)$ we have $K \subset A\left(\mathbb{R}^{n}\right)$ and $P \subset S-K \subset A\left(\mathbb{R}^{n}\right)$, so there are $u, v \in \mathbb{R}^{n}$ such that $y=A u$ and $z=A v$, hence $A x=A u+A v=$ $A(u+v)$ with $u \in A^{-1}(K)$ and $v \in A^{-1}(P)$. We thus have $A(x-u)=A v \in P$, which shows that $x-u \in A^{-1}(P)$, so $A^{-1}(S) \subset A^{-1}(K)+A^{-1}(P)$. The claim is then justified.

Let us now prove that $A^{-1}(K)=\left(A^{-1}(K)\right) \cap(\operatorname{Ker} A)^{\perp}+\operatorname{Ker} A$. For any $u \in\left(A^{-1}(K)\right) \cap(\operatorname{Ker} A)^{\perp}$ and $v \in \operatorname{Ker} A$, we have $A(u+v)=A u \in K$, thus $u+v \in A^{-1}(K)$. Conversely, let any $x \in A^{-1}(K)$. Writing $x=x_{A}+x_{\perp}$ with $x_{A} \in \operatorname{Ker} A$ and $x_{\perp} \in(\operatorname{Ker} A)^{\perp}$, we obtain $K \ni A x=A\left(x_{\perp}\right)$, hence $x_{\perp} \in\left(A^{-1}(K)\right) \cap(\operatorname{Ker} A)^{\perp}$. This gives $x \in\left(A^{-1}(K)\right) \cap(\operatorname{Ker} A)^{\perp}+\operatorname{Ker} A$, so the equality

$$
A^{-1}(K)=\left(A^{-1}(K)\right) \cap(\operatorname{Ker} A)^{\perp}+\operatorname{Ker} A
$$

holds true. Further, since $0^{+}\left(A^{-1}(K)\right) \subset \operatorname{Ker} A$, the convex set $\left(A^{-1}(K)\right) \cap$ $(\operatorname{Ker} A)^{\perp}$ is compact. To conclude that $A^{-1}(S)$ is M-decomposable it suffices to note that $A^{-1}(P)$ is a closed convex cone and to write, by (5) and by the equality established above for $A^{-1}(S)$, that

$$
\begin{aligned}
A^{-1}(S) & =\left(A^{-1}(K)\right) \cap(\operatorname{Ker} A)^{\perp}+\left(\operatorname{Ker} A+A^{-1}(P)\right) \\
& =\left(A^{-1}(K)\right) \cap(\operatorname{Ker} A)^{\perp}+A^{-1}(P),
\end{aligned}
$$

since Ker $A+A^{-1}(P)=A^{-1}(P)$ by the inclusion Ker $A \subset A^{-1}(P)$.

(b) Considering the linear mapping $T: \mathbb{R}^{n} \times \mathbb{R} \rightarrow \mathbb{R}^{m} \times \mathbb{R}$ defined by $T(x, r):=$ $(A x, r)$, we see that epi $(f \circ A)=T^{-1}($ epi $f)$. Therefore, (a) says that epi $(f \circ A)$ is M-decomposable, or equivalently the function $f \circ A$ is M-decomposable. 
Remark 13 Without the assumption $S \subset A\left(\mathbb{R}^{n}\right)$, the assertion (a) above may fail. Let $f: \mathbb{R}^{2} \rightarrow \mathbb{R}$ and $A: \mathbb{R}^{3} \rightarrow \mathbb{R}^{3}$ be defined for all $(x, y, z) \in \mathbb{R}^{3}$ by

$$
f(x, y):=\sqrt{(x-1)^{2}+y^{2}}=\|(x, y)-(1,0)\| \quad \text { and } \quad A(x, y, z):=(0, y, z),
$$

and let $S:=$ epi $f$. Considering the finite-valued convex function $h: \mathbb{R} \rightarrow \mathbb{R}$ defined by $h(y):=\sqrt{1+y^{2}}$ for all $y \in \mathbb{R}$, by easy computation (or see [6, $p$. 106]) one has $h^{*}\left(y^{*}\right)=-\sqrt{1-\left(y^{*}\right)^{2}}$ if $\left|y^{*}\right| \leq 1$ and $h^{*}\left(y^{*}\right)=+\infty$ otherwise, so $h^{*}$ is not Lipschitz on its domain. Corollary $8(d)$ tells us that $h$ is not $M$ decomposable, i.e., the set epi $h$ is not $M$-decomposable in $\mathbb{R}^{2}$. Since $A^{-1}(S)=$ $\mathbb{R} \times$ epi $h$, invoking Proposition 11 it follows that the set $A^{-1}(S)$ is not $M$ decomposable. Similarly, without the assumption $\operatorname{dom} f \subset A\left(\mathbb{R}^{n}\right)$, the assertion (b) above may fail. Let $f$ be the function we have just considered and $T: \mathbb{R}^{2} \rightarrow$ $\mathbb{R}^{2}$ be the linear mapping defined for all $(x, y) \in \mathbb{R}^{2}$ by

$$
T(x, y):=(0, y) .
$$

Noting that $f \circ T$ coincides with the function $h$ considered above, we see that $f \circ T$ is not $M$-decomposable while $f$ is.

Acknowledgments. J. E. Martínez-Legaz was partially supported by the MINECO of Spain, Grant MTM2014-59179- C2-2-P and the Severo Ochoa Programme for Centres of Excellence in R\&D [SEV-2015-0563]. He is affiliated with MOVE (Markets, Organizations and Votes in Economics).

\section{References}

[1] M.A. Goberna, E. González, J.E. Martínez-Legaz, M.I. Todorov, Motzkin decomposition of closed convex sets, J. Math. Anal. Appl. 364 (2010), 209221.

[2] M.A. Goberna, A.N. Iusem, J.E. Martínez-Legaz, M.I. Todorov, Motzkin decomposition of closed convex sets via truncation. J. Math. Anal. Appl. 400 (2013), 35-47.

[3] M.A. Goberna, J.E. Martínez-Legaz, M.I. Todorov, On Motzkin decomposable sets and functions, J. Math. Anal. Appl. 364 (2010), 525-537.

[4] A.N. Iusem, J.E. Martínez-Legaz, M.I. Todorov, Motzkin predecomposable sets, J. Glob. Optim. 60 (2014), 635-647.

[5] Th. Motzkin, Beiträge zur Theorie der Linearen Ungleichungen, Inaugural Dissertation 73 S., Basel, 1936.

[6] R.T. Rockafellar, Convex Analysis, Princeton University Press, Princeton, 1970. 